potential, predictable kinetics, drugs without renal or hepatic toxicity, and avoidance of GABAergic AEDs. (Shorvon S, Ferlisi M. The outcome of therapies in refractory and super-refractory convulsive status epilepticus and recommendations for therapy. Brain 2012 Aug;135(Pt 8):2314-28). (Respond: Dr Simon Shorvon, UCL Institute of Neurology, Queen Square, London WC1N 3BG, UK. E-mail: s.shorvon@ucl.ac.uk).

COMMENT. The authors comment that the most striking conclusion of their review of literature was the poor quality of outcome data. Only broad recommendations were possible from the analysis of reports. Refractory status epilepticus is heterogeneous and prognosis depends on factors other than treatment, such as age and etiology. General anesthesia is generally effective, and the rate of withdrawal seizures is lower than often quoted. Propofol infusion syndrome is a rare but frequently fatal complication caused by impaired fatty acid oxidation. The hallmarks are metabolic acidosis, lipemia, rhabdomyolysis and myocardial failure. A 10-year-old boy with status epilepticus treated with propofol developed fatal propofol infusion syndrome when a ketogenic diet was initiated. (Baumeister FA, et al. Neuropediatrics 2004 Aug;35(4):250-2).

\title{
LONG-TERM OUTCOME IN JUVENILE MYOCLONIC EPILEPSY
}

Researchers at University of Greifswald, Germany; and Cleveland Clinic, $\mathrm{OH}$, studied the long-term seizure outcome in patients with juvenile myoclonic epilepsy (JME) and identified factors predictive of seizure remission. Of 31 patients followed for at least 25 years (mean 39.1 years), $21(67.7 \%)$ were seizure-free, and $6(28.6 \%)$ had AEDs discontinued. Significant predictors for a poor long-term seizure outcome included occurrence of generalized tonic-clonic seizures (GTCS) preceded by bilateral myoclonic seizures $(\mathrm{p}=0.03)$, long duration of drug refractory epilepsy $(\mathrm{p}=0.022)$, and AED polytherapy $(\mathrm{p}=0.023)$. Complete remission of GTCS with AED significantly increases the chance for complete seizure freedom $(\mathrm{p}=0.012)$. Photoparoxysmal responses significantly increase risk of seizure recurrence after AED discontinuation $(\mathrm{p}=0.05)$. Long-term seizure freedom in two thirds of patients and validation of outcome predictors should permit clinicians to provide patients with a more favorable potential response to treatment. (Geithner J, Schneider F, Wang Z, et al. Predictors for long-term seizure outcome in juvenile myoclonic epilepsy: 25-63 years of follow-up. Epilepsia 2012 Aug;53(8):1379-86). (Respond: Dr Felix Schneider, Department of Neurology, Epilepsy Center, University of Greifswald, Sauerbruchstrasse, 17489 Greifswald, Germany. Email: felix.schneider@uni-greifswald.de).

COMMENT. A previous long-term study of JME in 24 patients, 25.8 years after seizure onset, found that $11(48 \%)$ had discontinued treatment and $6(25 \%)$ were seizurefree without AEDs for 5-23 years. (Camfield CS, Camfield PR. Neurology 2009 Sep 29;73(13):1041-5). Contrary to current opinion, these reports show that continuation of AED therapy in JME is not required in all patients, and predictive factors for long-term management and outcome are now available. 